\title{
DINSAR DEFORMATION TIME SERIES FOR MONITORING URBAN AREAS: THE IMPACT OF THE SECOND GENERATION SAR SYSTEMS
}

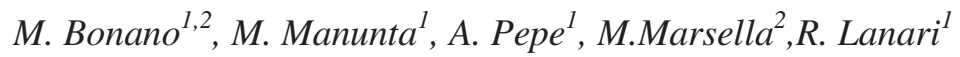 \\ ${ }^{1}$ IREA-CNR, 80124 Napoli, Italy \\ ${ }^{2}$ Università di Roma “La Sapienza”, Facoltà d'Ingegneria, DICEA, 00185, Roma, Italy
}

\begin{abstract}
We investigate the capability improvement of the DInSAR techniques to map deformation phenomena affecting urban areas, by performing a comparative analysis of the deformation time series retrieved by applying the full resolution Small BAseline Subset (SBAS) DInSAR technique to selected sequences of SAR data acquired by the ENVISAT, RADARSAT-1 and COSMO-SkyMed (CSK) SAR data.

The presented study, focused on the city of Napoli (Italy), allows us to quantify the dramatic increase of the DInSAR coherent pixel density achieved by exploiting the high resolution X-Band CSK SAR images with respect to the RADARSAT-1 and ENVISAT products, respectively; this permits us to analyze nearly all the structures located within the investigated urbanized area and, in many cases, also portions of a same building.
\end{abstract}

Index Terms -DInSAR, SBAS, COSMO-SkyMed, ENVISAT, RADARSAT-1, mapping capabilities, urban areas.

\section{INTRODUCTION}

Differential SAR Interferometry (DInSAR) has demonstrated over the last 20 years its capability to investigate several deformation phenomena, with few millimeter accuracy, over timescales ranging from weeks to decades, by analyzing the phase difference (interferogram) between two temporally-separated SAR images acquired over the same illuminated scene. Originally applied to investigate single deformation episodes, advanced DInSAR approaches have subsequently been developed to analyze the temporal evolution of the detected displacements through the generation of deformation time series. In this work, we focus on the advanced DInSAR technique referred to as
Small BAseline Subset (SBAS) approach [1], that implements an easy combination of DInSAR interferograms characterized by a small separation between the acquisition orbits (baseline), in order to mitigate the noise effects (decorrelation phenomena) thus maximizing the number of reliable measure pixels.

The SBAS technique allows investigating the detected ground displacements at two distinct spatial scales, referred to as regional and local, respectively [2]. At the regional scale the technique exploits average (multi-look) interferograms and permits to generate deformation velocity maps and corresponding time series relevant to very large areas (typically of about 100 x 100 $\mathrm{km})$. At the local scale, the technique exploits the singlelook interferograms, generated at full spatial resolution scale, in order to detect and analyze localized deformation phenomena affecting single buildings and man-made structures.

The SBAS algorithm has mostly been applied to SAR data acquired from 1992 to 2010 by the first-generation Cband $(5.6 \mathrm{~cm}$ wavelength) ERS-1/2 and ENVISAT sensors of the European Space Agency (ESA), with a nearly monthly revisit time and ground resolutions ranging from a few meters to tens of meters, but it has also been exploited to generate displacement time series from RADARSAT-1 SAR data of the Canadian Space Agency (CSA) [3], characterize by an improved spatial resolution of about $5 \mathrm{~m}$ ("Fine Beam" mode), although with a revisit time of 24 days. More recently, a "second generation" of SAR sensors has been launched (TerraSAR-X (TSX) and COSMO-SkyMed (CSK)). The growing availability of such SAR data collected at X-band (3.1 cm wavelength) characterized by improved spatial resolutions (1-3 m) and shorter revisit times (about 8 days for the CSK case) has encouraged us to adapt the SBAS-DInSAR technique to analyze X-band SAR data. 
In this work we investigate the improved capabilities of the advanced SBAS-DInSAR technique to analyze deformation phenomena affecting single buildings and man-made structures (or portions of them) in urban areas, by performing a comparative study of the deformation time series acquired by the new generation $\mathrm{X}$-Band sensors with respect to those of the first-generation CBand systems. In particular, we have applied the full resolution SBAS approach [2] to three SAR datasets collected over the city of Napoli (Italy) by the ASAR/ENVISAT, RADARSAT-1 and COSMO-SkyMed sensors, respectively. Furthermore, since some of the SAR images acquired by the CSK radar sensors are characterized by a significant Doppler Centroid (DC) offset, we have also exploited the procedure presented in [4] to retrieve information about the sub-pixel azimuth location of the coherent CSK SAR pixels.

The achieved results demonstrate the drastic improvement gained by the advanced DInSAR techniques when exploiting X-band SAR data, allowing us to analyze nearly all the structures located within the investigated urban area.

\section{FULL RESOLUTION SBAS ALGORITHM}

Let us consider a set of $N+1$ SAR images, properly coregistered to a reference master image. From this image series, two set of multi-look (i.e., Low-Pass filtered) and single-look differential interferograms are generated, by constraining their maximum spatial and temporal baseline values as well as their Doppler Centroid difference.

The extended full resolution SBAS analysis starts with the modulo- $2 \pi$ subtraction of the Low Pass phase signals, retrieved by the multi-look interferograms, from the single-look interferograms [2], leading to an estimate, on a pixel by pixel basis, of the High-Pass (HP) interferometric phase components, which need to be properly unwrapped. The unwrapping operation is carried out, for each pixel, by first estimating a linear phase model [4], which depends on topography $\Delta z$ and deformation velocity $\Delta v$ high-pass phase components as well as the point scatterer azimuth location $\Delta x$, and maximizes the temporal coherence [2]; then subtracting from the HP phase component the computed linear phase model, in correspondence to the pixels where the temporal coherence value is greater than a selected threshold. This subtraction allows identifying the non-linear phase component, which is assumed to be within the $(-\pi, \pi)$ interval, thus avoiding any additional unwrapping operation. The overall deformation signal can be easily computed by combining the displacements from the multilook signals and the linear and non-linear HP signal components.
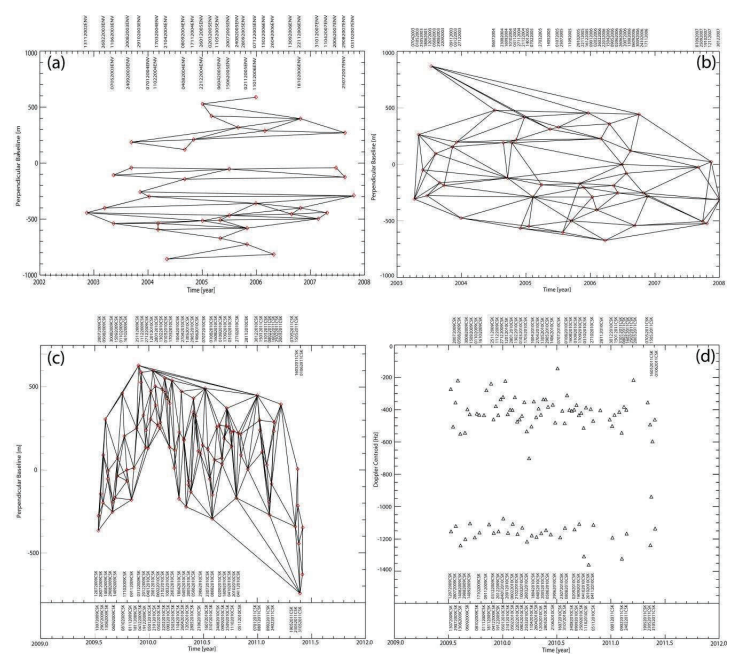

Figure 1 - (a) ENVISAT, (b) RADARSAT-1 and (c) COSMO-SkyMed SAR data distribution in the temporal/perpendicular baseline plane relevant to the Napoli Bay area. The red diamonds in (a), (b) and (c) represent the acquisitions while each arc of the graph corresponds to a computed interferogram. (d) SAR data representation in the temporal baseline/DC plane for the CSK dataset; the black triangles in (d) identify the CSK acquisitions.

\section{DInSAR PRODUCTS RETRIEVED VIA SBAS INVERSION}

We have applied the extended full resolution SBAS algorithm [4] to three archives of SAR data acquired over the city of Napoli (Italy) by the ENVISAT, RADARSAT1 and CSK radar systems, respectively. In particular, the ENVISAT dataset consists of 37 SAR images (November 2002-October 2007, IS2 swath); the RADARSAT-1 archive is composed by 47 SAR images from April 2003 to December 2007 through the Fine Beam mode, whereas the CSK dataset consists of 99 data frames collected over the time period from July 2009 to June 2011. The available SAR datasets have been paired to form three corresponding sequences of small baseline interferograms, identified by imposing constraints on the maximum allowed perpendicular baseline values. The distributions of the selected interferometric SAR acquisitions in the temporal/perpendicular baseline plane for the ENVISAT, RADARSAT-1 and CSK datasets are pictorially represented in Figures 1(a)-(c), respectively. In addition, to stress the offset that characterizes the DC values of some of the CSK SAR acquisitions, we also show in Figure 1(d) the representation of the CSK dataset within the temporal baseline/DC domain, where it is evident the presence of a $800 \mathrm{~Hz}$ DC offset for a subset of the CSK acquisitions. For the interferogram generation, precise 

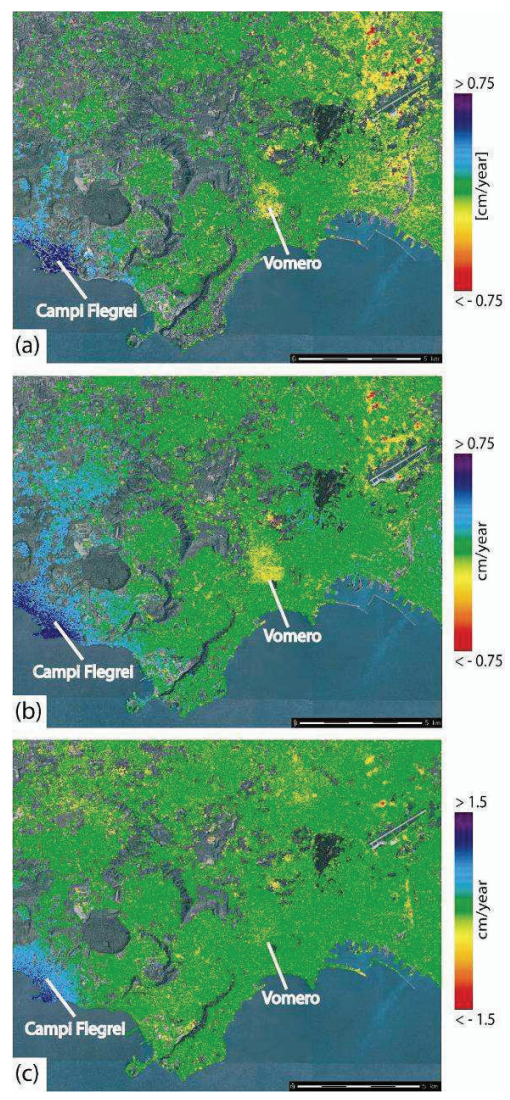

Figure 2 - Geocoded full resolution deformation velocity maps superimposed on an orthophoto of the Napoli Bay area (Italy). The results are relevant to the (a) ENVISAT, (b) RADARSAT-1 and (c) CSK SAR datasets, respectively. The Campi Flegrei caldera and the Vomero quarter, within the city of Napoli, have been highlighted.

orbital information and the three-arcsec DEM of the study area obtained by the Shuttle Radar Topography Mission (SRTM) have been used to estimate the topographic phase component.

To provide an overall view of the detected deformation affecting the Napoli Bay area, we present in Figures 2(a)-(c) the retrieved geocoded mean deformation velocity maps, superimposed on an orthophoto of the investigated zone. Figures 2(a)-(c) clearly show that some significant displacement patterns, easily identified on each deformation velocity map, are present in several areas, although characterized by different deformation rates due to the different time span of each SAR dataset.

To perform a quantitative assessment of the DInSAR mapping improvement achieved in urban areas, we have carried out a comparative analysis on the DInSAR products relevant to the Napoli centre. In particular, to
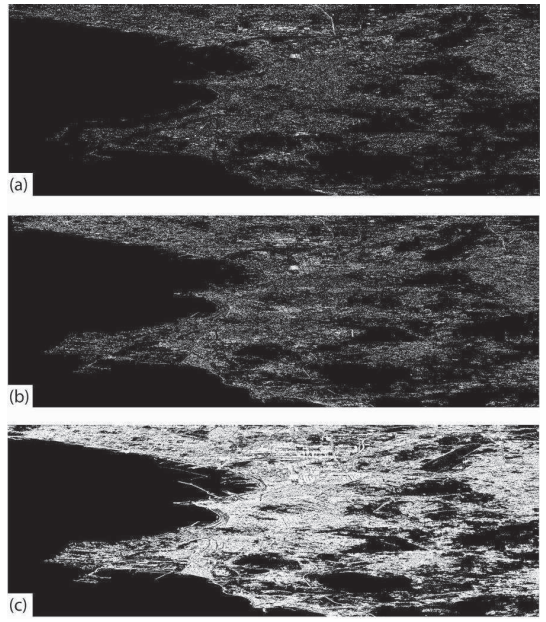

Figure 3 - Maps of coherent pixels identified by processing the available datasets acquired by the (a) ENVISAT, (b) RADARSAT-1 and (c) CSK SAR sensors over the urbanized area of Napoli. The coherent points detected through the three different radar systems are represented by white pixels onto the reference azimuth/range spatial grid of the ENVISAT system.

achieve a correct estimate about the increased spatial density of coherent pixels, as we move from the ENVISAT and RADARSAT- 1 results to the CSK ones, we have computed the map of coherent pixels retrieved through the ENVISAT radar imaging, which is shown in Figure 3(a). Subsequently, we have properly identified the position of the coherent pixels imaged by the RADARSAT-1 [Figure 3(b)] and CSK [Figure 3(c)] sensors with respect to the ENVISAT azimuth/range spatial grid, taken as reference. We have obtained a spatial density of about 650 points $/ \mathrm{km}^{2}$ for the ENVISAT map [Figure 3(a)], whereas in the RADARSAT-1 case the detected spatial density increases to about 1000 points $/ \mathrm{km}^{2}$ [Figure 3(b)], reasonably due to the improved spatial resolution of the RADARSAT-1 system with respect to ENVISAT one, being the operational wavelength, the overall time span and the number of acquisitions of the two SAR datasets rather similar. By moving to CSK SAR datasets, our analysis has revealed a spatial density of the coherent pixels of about 4200 points $/ \mathrm{km}^{2}$, which corresponds to a percentage increase of about $320 \%$ and $550 \%$ with respect to the RADARSAT-1 and ENVISAT cases, respectively.

In order to highlight the impact of such a drastic increase of coherent pixels, let us consider a key example relevant to the zone nearby the Napoli Central Railway station, see Figures 4(a)-(c), which has been interested by tunnel excavation operations since 2005 for the setting-up of the new metro line (red paths). It is clear that as we move from the ENVISAT results (top) to those of the 
RADARSAT-1 data (middle) and then to the CSK ones (bottom), the impact of the increase of the coherent pixel density is impressive, as for the case of the roof of the railway station identified by the black arrow in Figure 4(c). Moreover, the three velocity maps of Figure 4 clearly indicate that the detected deformation signals have rather different rates due to the three distinctive time periods of observation. We analyze the deformation time series retrieved by the ENVISAT, RADARSAT-1 and CSK radar sensors plotted in Figures 4(d)-(f), respectively, computed in correspondence to the three pixels, located in proximity of the area involved in the new tunnel excavation, labeled to as $\mathrm{P}_{1}, \mathrm{P}_{2}$ and $\mathrm{P}_{3}$ in Figures 4(a)-(c), respectively. It is worth to remark that the computed ENVISAT and RADARSAT-1 deformation time series allow the detection of the subsidence phenomena that have affected the zone since 2005. On the other hand, the CSK time series of Figure 4(f) evidences an uplift deformation episode, which is very likely related to the consolidation activity carried out in this zone at the end of the excavation works. This latter example is particularly effective because it also allows us to clearly show the impact of the high temporal sampling rate of the CSK deformation time series (nearly 100 images area available within a two-year time interval), with respect to ENVISAT and RADARSAT-1, which is beneficial for the detection and monitoring of such a small and fast-varying deformation signal.

\section{REFERENCES}

[1] Berardino, P., Fornaro, G., Lanari, R. and Sansosti,E. "A new algorithm for surface deformation monitoring based on small baseline differential SAR interferograms," IEEE Trans. Geosci. Remote Sens., vol. 40, no. 11, pp. 2375-
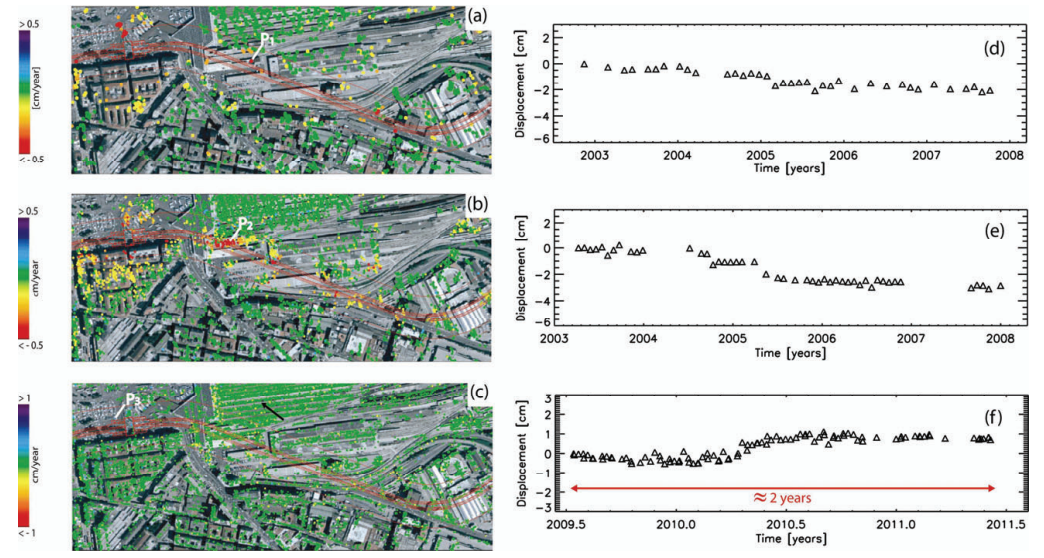

Figure 4 - Geocoded mean deformation velocity maps computed by processing the (a) ENVISAT, (b) RADARSAT-1 and (c) CSK data archives, related to the area nearby the Napoli Central Railway station. The red line superimposed on the three maps represents the trace of the new metro line excavated tunnels, whereas the black arrow identifies the roof of the railway station. (d), (e), (f) Plots of the computed deformation time-series relevant to the pixels labelled to as P1, P2 and P3 in (a), (b) and (c), respectively. 